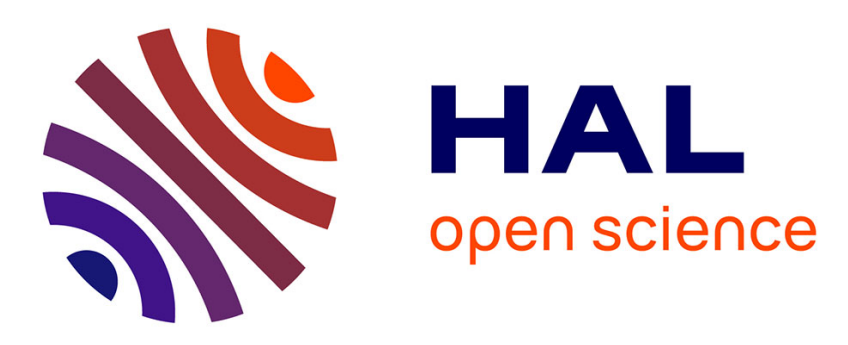

\title{
Compact multichannel infrared camera integrated in an operational detector dewar cooler assembly
}

\author{
Florence de La Barrière, Guillaume Druart, Nicolas Guérineau, Frédéric
}

Champagnat, Aurélien Plyer, Gilles Lasfargues, Serge Magli

\section{- To cite this version:}

Florence de La Barrière, Guillaume Druart, Nicolas Guérineau, Frédéric Champagnat, Aurélien Plyer, et al.. Compact multichannel infrared camera integrated in an operational detector dewar cooler assembly. Applied optics, 2018, 57 (17), pp.4761-4770. 10.1364/AO.57.004761 . hal-01831691

\section{HAL Id: hal-01831691 \\ https://hal.science/hal-01831691}

Submitted on 6 Jul 2018

HAL is a multi-disciplinary open access archive for the deposit and dissemination of scientific research documents, whether they are published or not. The documents may come from teaching and research institutions in France or abroad, or from public or private research centers.
L'archive ouverte pluridisciplinaire HAL, est destinée au dépôt et à la diffusion de documents scientifiques de niveau recherche, publiés ou non, émanant des établissements d'enseignement et de recherche français ou étrangers, des laboratoires publics ou privés. 


\title{
Compact multichannel infrared camera integrated in an operational detector dewar cooler assembly
}

\author{
Florence de la Barrière ${ }^{1, *}$, Guillaume Druart ${ }^{1}$, Nicolas GuérineaU ${ }^{1}$, Frédéric \\ Champagnat $^{2}$, Aurélien Plyer ${ }^{2}$, Gilles lasfargues ${ }^{3}$, and Serge Magli ${ }^{4}$ \\ ${ }^{1}$ DOTA, ONERA, Université Paris-Saclay, F-91123 Palaiseau, France \\ ${ }^{2}$ DTIS, ONERA, Université Paris-Saclay, F-91123 Palaiseau, France \\ ${ }^{3}$ CEA LETI MINATEC, 17 rue des Martyrs, F-38054 Grenoble, France \\ ${ }^{4}$ SOFRADIR, 364 route de Valence - Actipole - CS 10021, F-38113 Veurey-Voroize, France \\ *Corresponding author: florence.de_la_barriere@onera.fr
}

Compiled June 1, 2018

\begin{abstract}
We present an ultra-compact infrared cryogenic camera integrated inside a standard SOFRADIR's Detector Dewar Cooler Assembly (DDCA) and whose field of view is equal to $120^{\circ}$. The multichannel optical architecture produces four non-redundant images on a single SCORPIO detector with a pixel pitch of $15 \mu \mathrm{m}$. This ultra-miniaturized optical system brings a very low additional optical and mechanical mass to be cooled in the DDCA: the cool-down time is comparable to the one of an equivalent DDCA without an imagery function. Limiting the number of channels is necessary to keep the highest number of resolved points in the final image. However, optical tolerances lead to irregular shifts between the channels. This paper discusses the limits of multichannel architectures. With an image processing algorithm, the four images produced by the camera are combined to process a single full-resolution image with an equivalent sampling pitch equal to $7.5 \mu \mathrm{m}$. Experimental measurements on MTF and NETD show that this camera achieves good optical performances. (c) 2018 Optical Society of America
\end{abstract}

OCIS codes: (110.3080) Infrared imaging; (220.4830) Systems design; (110.4190) Multiple imaging; (110.4155) Multiframe image processing; (350.3950) Micro-optics.

\section{INTRODUCTION}

Nowadays, military and civilian applications require miniaturized optical systems. Optical architectures have to be compatible with a very low budget in terms of mass, volume and power consumption. These constraints are so demanding that downscaled versions of traditional optical systems with a single optical axis are reaching their limits because of a loss in the number of resolved points in the image [1]. Inspired by the compound eyes of invertebrates [2], multichannel imaging seems to be a promising approach to address this issue. Several types of multichannel architectures have been proposed. Two different approaches can be distinguished. On the one hand, each channel captures a low-resolution image of the overall field of view (FOV), which is known as the thin observation module by bound optics (TOMBO) principle [3-7]. If the spectral content of the subimages is non-redundant [8], super-resolution techniques can be applied to retrieve the high frequency content of the scene [3,9-14]. On the other hand, each channel images a different part of the scene with a reduced FOV [15-25]. The optical axes of the different channels are tilted with respect to each other. The images are then stitched together with an image processing technique in order to reconstruct a final image. Most of the concepts cited above operate in the visible spectral range, and only few address the infrared spectral range (refs. [4, 5, 7] work in the long-wave infrared (LWIR) band).

Our field of interest addresses cooled infrared detectors working in the mid-wave infrared (MWIR) band. Infrared focal plane arrays (IRFPA), based on HgCdTe technology, are very sensitive detectors which need to be cooled at a temperature around $90 \mathrm{~K}$. That is why they are integrated in a Detector Dewar Cooler Assembly (DDCA), which is a vacuum chamber closed by a window. A cold shield and a cold diaphragm limit the angle of view of the IRFPA, in order to avoid background emission from the external roof of the Dewar at ambient temperature. Our work aims at miniaturizing optical systems associated with cooled IRFPAs; to do that, we have to integrate the optics directly inside the DDCA. The Dewar is a sealed and cooled environment traditionally dedicated to the IR detector. Specific constraints are linked to the demanding cooled environment: the cryogenic optical architecture has to be as simple as possible and it must have a minimal size. Moreover, the op- 
tical and mechanical mass added in the cold shield has to be limited, so that the cool-down time could be comparable to the one of a traditional DDCA without optical parts, and the system could remain compatible with operational constraints.

Our approach first consists in integrating a very simple system in the cold shield: it relies on a traditional lens which images the scene with a good optical quality [26]. The miniaturization process can be extremely emphasized by integrating the optical system directly on the IRFPA. To achieve extreme compactness, several design rules can be applied: a detailed description of these design strategies can be found in a previous paper [27]. Miniaturization can be achieved by designing multichannel optical systems. We made a cryogenic wafer-level camera whose total track length is equal to $4.08 \mathrm{~mm}$ only [28]. However, it is associated with a laboratory IRFPA, and integrated in a laboratory Dewar. Our goal is to adapt the previous wafer-level design to the constraints of a SCORPIO detector integrated in an operational DDCA commercialized by SOFRADIR [29]. In a previous paper [30], we briefly described a new optical and mechanical design which is adapted to a commercial DDCA. In section 2 of this paper, we give more details about the optical design of this prototype. In section 3, we show the optomechanical design. In section 4 , we measure the distortion by scanning a point source in front of the camera. The distortion measurements reveal that the shifts between the sub-images differ from the theoretical expected ones, due to technological constraints. We present a new image processing method, called SR Viewer, described in section 5, which is more adapted to the camera than the traditional "shift and add" method. We also simulate errors in the optical architecture to show how irregular sampling can be predicted. We measure the MTF with the bar targets method, with frequencies higher than the Nyquist frequency, which illustrates the quality of the SR Viewer image processing. By controlling the whole image formation chain, from optical design to image processing, this paper addresses the interests and the limitations of multichannel systems.

\section{OPTICAL DESIGN OF THE COMPACT INFRARED CAMERA}

\section{A. Starting point with a single lens}

We have to limit the additionnal mass to be cooled in order not to increase the cool-down time. Thus, as a starting point, we want to design a simple infrared camera made of a single lens integrated in the Dewar. We want to use a SCORPIO off-theshelf detector [29], which has the following features : $640 \times 512$ pixels, a sampling pitch equal to $15 \mu \mathrm{m}$ and a fill factor equal to 1 . The field of view will be equal to $120^{\circ}$ on the diagonal of a $512 \times 512$ pixels detector (i.e. $10.9 \mathrm{~mm}$ ). To assure correct radiometric performances, we choose a $f$-number equal to 3 .

The paraxial focal length is equal to $3.1 \mathrm{~mm}$. In practise, to limit astigmatism, the pupil has to be moved away from the lens instead of being on it. After optimization with the Zemax optical software, we obtain the landscape design illustrated in Figure 1, with an entrance pupil in front of the lens. Its main characteristics are given in Table 1 . The surface in front of the detector is conic and diffractive (in order to correct lateral chromatic aberration). The lens is made of Germanium. Its thickness on the optical axis is equal to $1.5 \mathrm{~mm}$.

For manufacturing purposes, and to be able to use thin optics, we want to keep the lens as simple as possible. That is why only one complex surface (conic and diffractive) is allowed, the other is kept spheric. The modulation transfer function (MTF)

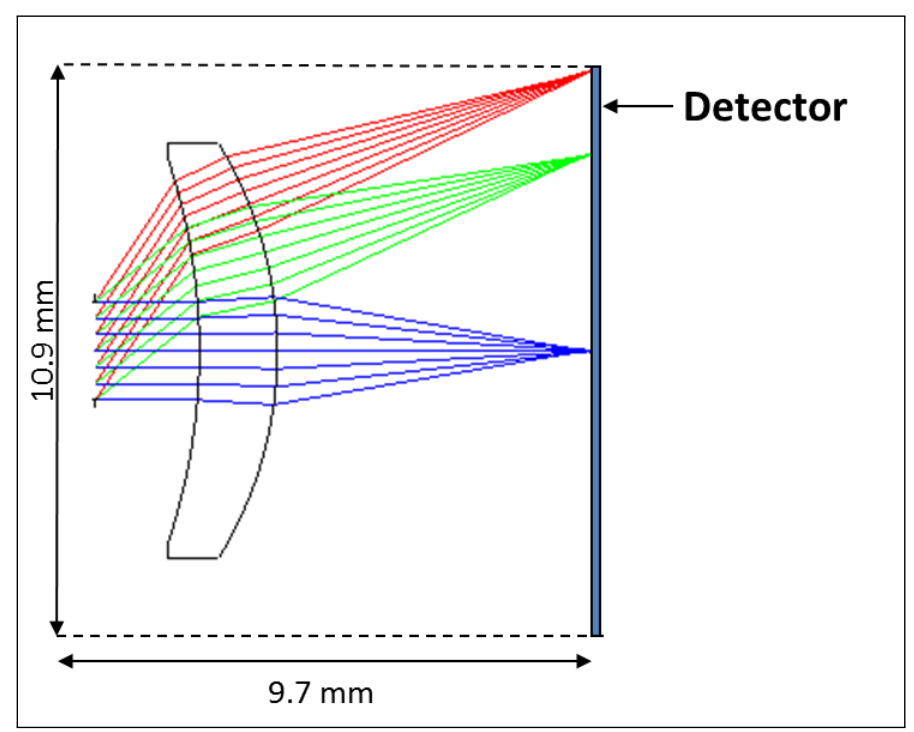

Fig. 1. Optical layout of the starting point using a single lens. The different colors stand for different field angles.

Table 1. Optical characteristics of the simple infrared camera based on a single lens illustrated in Figure 1.

\begin{tabular}{ll}
\hline Property & Value \\
\hline $\begin{array}{l}\text { Size of optics module }(\mathrm{L} \times \mathrm{W} \times \\
\mathrm{T})\end{array}$ & $10.9 \mathrm{~mm} \times 10.9 \mathrm{~mm} \times 9.7 \mathrm{~mm}$ \\
Field of view & $120^{\circ}$ \\
$f$-number & 3 \\
Focal length & $5.9 \mathrm{~mm}$ \\
Number of pixels & $512 \times 512$ (diagonal) \\
Clear diameter of lens & $7.4 \mathrm{~mm}$ \\
Pinhole diameter & $2 \mathrm{~mm}$ \\
Optical mass of the lens & $0.346 \mathrm{~g}$ \\
\hline
\end{tabular}


of the optical system is illustrated in Figure 2, until the Nyquist frequency of a focal plane array with a sampling pitch equal to $15 \mu \mathrm{m}$, i.e. $33.3 \mathrm{cy} / \mathrm{mm}$ (the MTF of the pixel is not taken into account). The optical system is not limited by the diffraction: it is not so easy to correct optical aberrations while keeping the system as simple as possible.

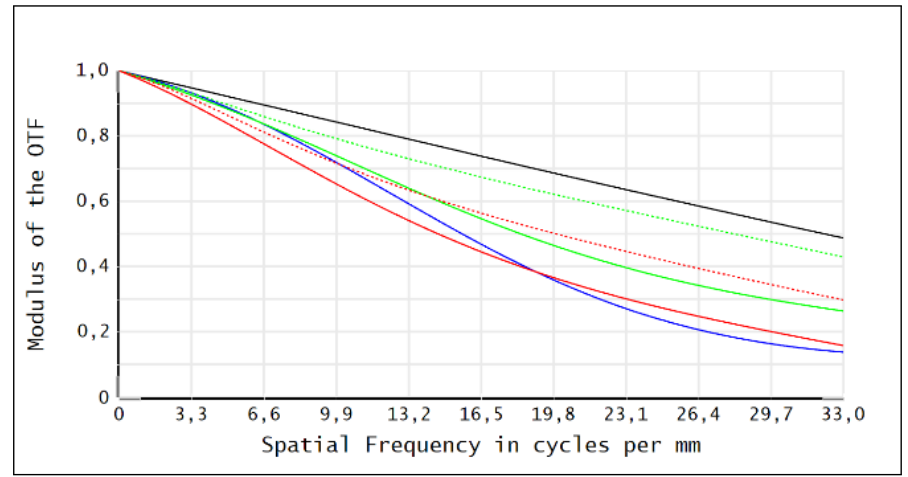

Fig. 2. MTF of the optical system illustrated in Fig. 1, extracted from Zemax, for different field points (the different colors stand for different values of the FOV). Black curve: diffraction limit, blue: MTF on axis, green: MTF for 7.6mm FOV tangential, green dashed: MTF for $10.9 \mathrm{~mm}$ FOV sagittal, red: MTF for $10.9 \mathrm{~mm}$ FOV tangential, red dashed: MTF for $5.43 \mathrm{~mm}$ FOV sagittal.

We want to improve this starting point by looking for a system which would have a better optical quality while still relying on a single optical component.

\section{B. Design of a compact multichannel camera}

One of the strategies to achieve extreme compactness consists in decreasing the focal length of the optical system [27]. In order to compensate for the loss of resolved points, replicating an undersampled miniaturized system while providing a subpixel shift between adjacent channels is an efficient solution. We still want to use the SCORPIO detector. In the following, we are going to determine how many channels are necessary to retrieve information with an image processing method, called superresolution. In this paper, what we mean with super-resolution is reducing the sampling pitch, which allows maintaining the number of resolved points with theoretical shifts. For cooled MWIR detectors made in $\mathrm{HgCdTe}$, the diffusion length of the carriers [31] reduces the MTF of the pixel, as illustrated in Figure 3. Figure 3 represents (i) the MTF of a $15 \mu \mathrm{m}$ pitch square pixel, it is a sinus cardinal with its first zero at spatial frequency $66.6 \mathrm{cy} / \mathrm{mm}$, (ii) the MTF due to the diffusion length of the carriers, which is a Lorentz function, and (iii) the product of the two latest MTF. The global MTF after the cut-off frequency is reduced by a factor 2.9 (from 0.215 without diffusion to 0.075 with diffusion). Trying to retrieve frequencies higher than the pixel cut-off frequency is not so interesting because the lobes have a small amplitude. As we only want to retrieve frequencies up to twice the Nyquist frequency, only $2 \times 2$ channels are necessary. Moreover, in order to use the maximum pixels available on the SCORPIO IRFPA, the best solution is to have only the theoretical necessary number of channels $(2 \times 2)$, and not to increase this number to avoid losing resolved points in the final processed image.

The subpixel shift between two adjacent images has to be

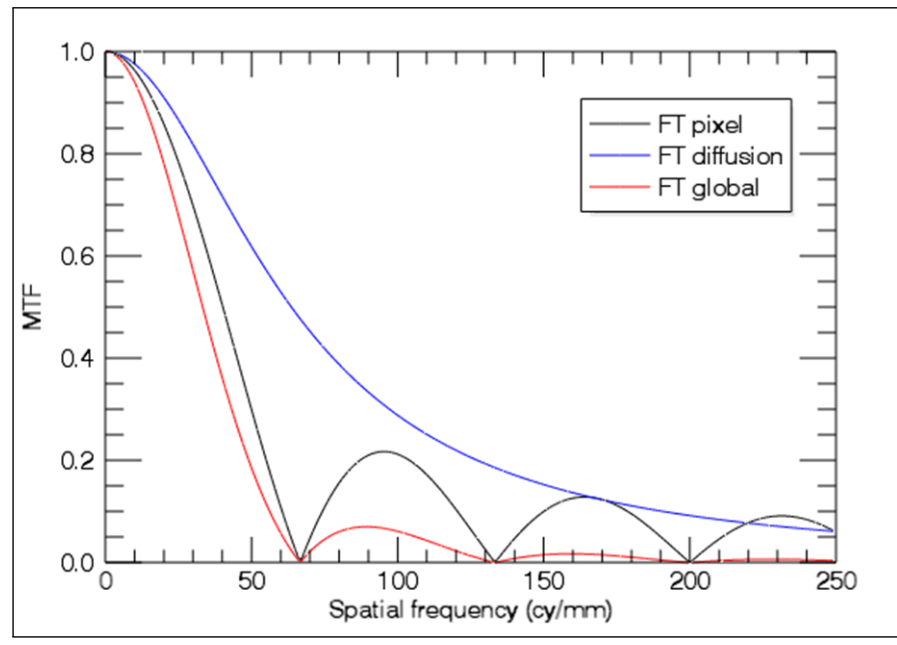

Fig. 3. Simulation of the pixel MTF for a pixel pitch equal to $15 \mu \mathrm{m}$ and a fill factor equal to 1 , the MTF due to the diffusion length of carriers in $\mathrm{HgCdTe}$, and the global MTF. The global MTF contains poor information after the cut-off frequency of the pixel (66cy/ $\mathrm{mm}$ here).

equal to half the sampling pitch $(7.5 \mu \mathrm{m})$, so that the equivalent sampling pitch of the final image is $7.5 \mu \mathrm{m}$ (which corresponds to a Nyquist frequency of $66 \mathrm{cy} / \mathrm{mm}$ ). This value is lower than the smallest sampling pitch which is commercialized nowadays $(10 \mu \mathrm{m})$ [29]. Each channel is associated with $256 \times 256$ pixels of the IRFPA, and the final processed image is expected to have $512 \times 512$ resolved points. A cold filter limits the bandwidth of the camera to $[3.7 \mu \mathrm{m}-4.8 \mu \mathrm{m}]$.

\section{Impact of technological constraints on optical design}

As we merge the optics and the detector to make an ultracompact camera, technological constrains linked to manufacturing and integrating the optical module near the detector have to be taken into account while designing the system.

In previous work [28], the wafer-level camera is totally integrated on the IRFPA: it is made of two stages of Silicon lenslet arrays. The distances between the two lenslet arrays and between the optical module and the detector are equal to a few hundreds of micrometers. The opto-mechanical assembly relies on an innovative way to integrate the optical module directly on the ceramic of the detector. Indeed, Indium bumps are used as spacers between the two stages of lenslet arrays and between the detector and the optical module.

In the current optical design, the detector format increases by a factor of four compared with the one used in the previous demonstrator. Therefore, the focal length of the optics doubles and the back focal length increases too. The distances between some optical components and the detector are greater than $700 \mu \mathrm{m}$ (this technological limit is mentioned in previous work [28]) even if we place the cold filter at equal distance between the detector and the array of lenses. Using Indium bumps as spacers can no longer be applied and the opto-mechanical design needs to be adapted: we use the cold shield as an optical mount, as explained in Section 3.

As the optical architecture is integrated in the cold shield near the detector, it has to be adapted to thermo-mechanical constraints. A study of the SCORPIO detector shows that Germanium has better properties to fulfil the latest requirement 
than Silicon. Therefore, we choose Germanium as the optical material of the lens. We carry out an optimization process on a single channel using Zemax. The optical layout of a single channel is illustrated in Figure 4. It is simply made of a pinhole and a Germanium plano-convex lens, whose curved face has an aspheric profile. The camera is ultra-compact, since its total track length is lower than $3.8 \mathrm{~mm}$. Table 2 sums up the optical characteristics of the multichannel system. Figure 5 illustrates the optical MTF (the pixel MTF is not taken into account). By comparing with Figure 2, we can notice that the optical quality is better than the system with a single lens, as the design is nearly limited by the diffraction.

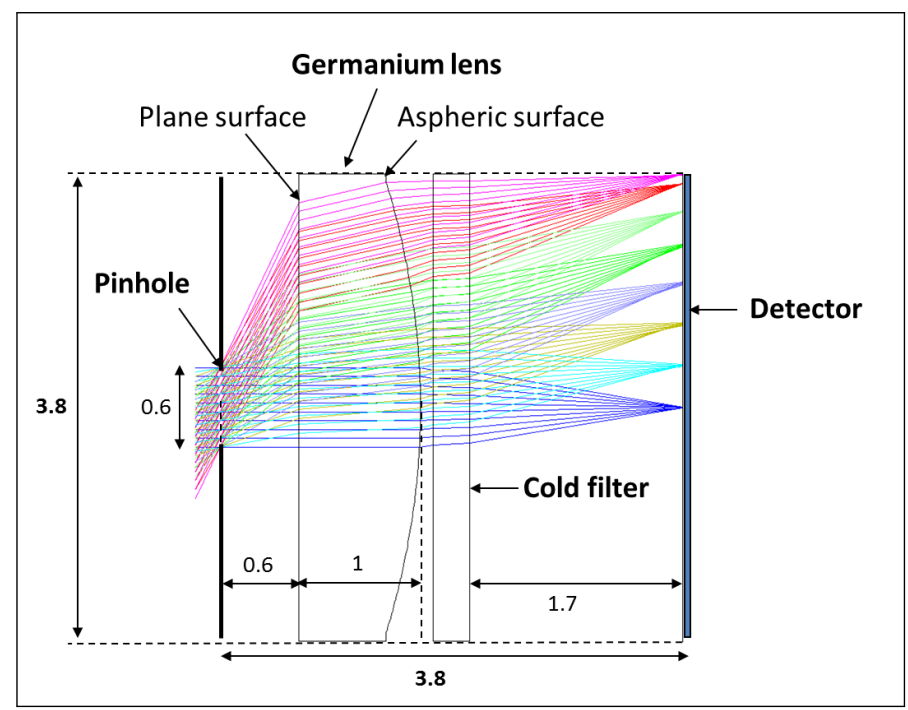

Fig. 4. Optical layout of one channel of the ultra-thin infrared camera. The different colors stand for different field angles. Length units are $\mathrm{mm}$.

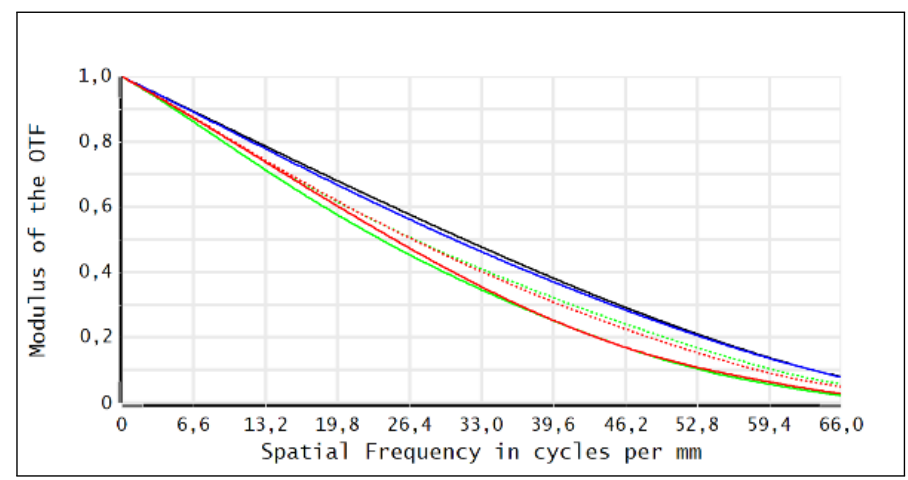

Fig. 5. MTF of the optical system illustrated in Fig. 4, extracted from Zemax, for different field points (the different colors stand for different values of the FOV). Black curve: diffraction limit, blue: MTF on axis, green: MTF for $45^{\circ}$ FOV tangential, green dashed: MTF for $45^{\circ}$ FOV sagittal, red: MTF for $120^{\circ}$ FOV tangential, red dashed: MTF for $120^{\circ}$ FOV sagittal.

The optical design could be improved in the following ways to reduce the optical mass in the cold shield. Firstly, a thinner plano-convex lens (less than $1 \mathrm{~mm}$ ) could be obtained by using high-order Fresnel lenses, as shown in previous work [32]. Secondly, the cold filter may be deposited directly on the plane
Table 2. Optical characteristics of the infrared multichannel camera based on the channel illustrated in Figure 4.

\begin{tabular}{|c|c|}
\hline Property & Value \\
\hline $\begin{array}{l}\text { Size of optics module }(\mathrm{L} x \mathrm{~W} x \\
\mathrm{T})\end{array}$ & $\begin{array}{l}\text { lower than } 8 m m \times 8 m m \times \\
6 m m\end{array}$ \\
\hline Field of view & $120^{\circ}$ \\
\hline$f$-number of one channel & 3 \\
\hline Focal length of one channel & $1.96 \mathrm{~mm}$ \\
\hline Number of channels & $2 \times 2$ \\
\hline $\begin{array}{l}\text { Thickness of small lenses on the } \\
\text { optical axis }\end{array}$ & $1 \mathrm{~mm}$ \\
\hline Number of pixels per channel & $256 \times 256$ \\
\hline lenslet array pitch & $3.8 \mathrm{~mm}$ \\
\hline Pinhole diameter & $0.6 \mathrm{~mm}$ \\
\hline Optical quality & Limited by the diffraction \\
\hline Shift between adjacent channels & $7.5 \mu m$ \\
\hline $\begin{array}{l}\text { Expected number of resolved } \\
\text { points after image processing }\end{array}$ & $512 \times 512$ \\
\hline $\begin{array}{l}\text { Mass of the optical component } \\
\text { (lenslet array) }\end{array}$ & lower than $0.7 g$ \\
\hline
\end{tabular}

surface of the lens (the difficulty may be that the rays for the maximal field of view angle have a high angle of incidence on the plane surface).

If we used the Scorpio detector with a single channel system (as described in section 2A with the starting point), we would obtain a single image on $640 \times 512$ pixels, sampled with a pitch equal to $15 \mu \mathrm{m}$ (pixel pitch of the detector). With our multichannel design, after image processing such as superresolution, and if the system was perfect, we would obtain a single image with $512 \times 512$ pixels with an equivalent sampling pitch of $7.5 \mu \mathrm{m}=15 \mu \mathrm{m} / 2$. It would be possible to obtain such resolution with a single channel optical system only if we had infrared detectors with a pitch of $7.5 \mu \mathrm{m}$, which is not the state-of-the-art today at Sofradir.

\section{FABRICATION OF THE INFRARED ULTRA-THIN CAM- ERA}

The Germanium lenslet array was fabricated using diamondturning techniques, which enable the manufacturing of the aspheric profile and of the multichannel design. The manufacturing has been carried out by the French company Savimex [33]. Then, an anti-reflective coating has been deposited on the lenslet array.

We have adapted the design of the cold shield in order to integrate the optical elements (pinhole array, lenslet array and cold filter) near the detector. Small walls have been provided at the top of the cold shield to limit rays with an incident angle greater than the field of view $\left(120^{\circ}\right)$. The opto-mechanical design is illustrated in Figure 6. The overall height of the cold shield is lower than $6 \mathrm{~mm}$, whereas the height of traditional cold shields is between $20 \mathrm{~mm}$ and $30 \mathrm{~mm}$. The mass of the 
optical component (lenslet array with its optical and mechanical parts) is lower than $0.7 \mathrm{~g}$. Thus, the lenslet array is heavier than the single lens of Fig. 1, but the additional optical mass is nearly compensated by the reduced mechanical mass, as the cold shield is less high. The cool-down time of the camera is comparable to the one of an equivalent DDCA without an imagery function: it is equal to 6 minutes with a THALES Cryogenics RM3. Thus, the camera remains fully compatible with operational constraints. Designing a simple optical system (based on a pinhole array and a lenslet array only) and integrating it in an adapted cold shield leads to a very low additional optical and mechanical mass to be cooled.

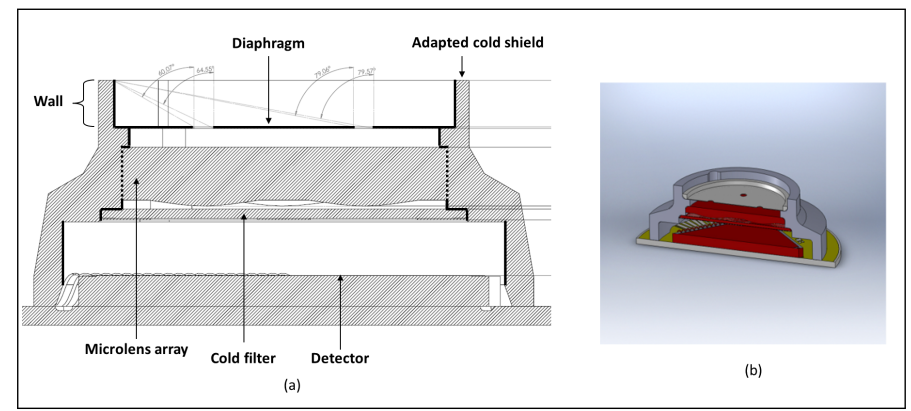

Fig. 6. (a) Opto-mechanical design of the cryogenic ultra-thin camera, (b) Section of the cold shield.

Then, the whole system has been integrated in an operational DDCA commercialized by SOFRADIR: it is illustrated in Figure 7.

\section{CHARACTERIZATION OF THE ULTRA-THIN CRYO- GENIC INFRARED CAMERA}

We performed MTF and NETD measurements. Details on the measurements method and on results can be found in previous work [30]. The main conclusions are that the four channels have similar MTF curves, which means that they are identical from an optical point of view. The quality of the optical system is almost limited by the diffraction, as expected from the theoretical study. The NETD of the whole camera (detector and optics) is equal to $17 \mathrm{mK}$ for $50 \%$ well-fill and a scene at $293 \mathrm{~K}$, which is close to the NETD of the detector alone. The background current due to the instrument is very low; indeed, we measure a very low background current, around $3 p A$ only, because the whole optical system is cooled. In this section, we go deeper in the characterization of the miniaturized infrared camera. We measured the relative illumination in the field and the distortion.

\section{A. Relative illumination in the field}

We illuminate the Dewar window with a flat blackbody at $293 \mathrm{~K}$ : we obtain the image illustrated in Figure 8. We can clearly distinguish the four channels of the camera. We plot the normalized illumination as a function of height in the image plane. We compare this graph to the theoretical data extracted from the Zemax software, as shown in Figure 9. For each subimage, the illumination decreases from the center to the edge, as expected. This evolution is the same for the four channels. We can notice that the decrease is more important on the measured data than on the theoretical one: the decrease on the experimental data is equal to $30 \%$ at $1.5 \mathrm{~mm}$ from the center of the channel, whereas

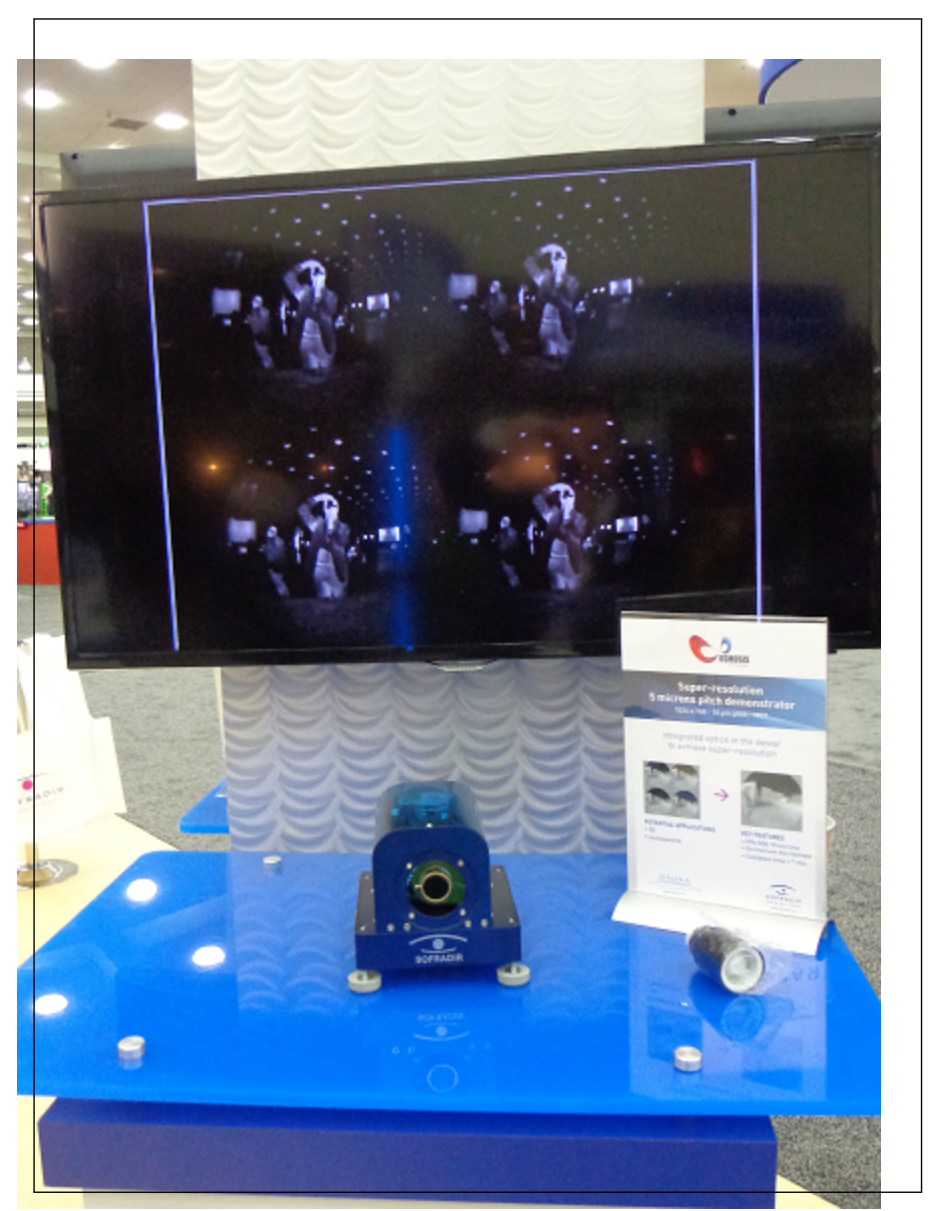

Fig. 7. Photograph of the ultra-thin infrared camera integrated in an operational DDCA commercialized by SOFRADIR. 
it is only equal to $20 \%$ for the same point on the theoretical data. This difference may be explained by Fresnel losses linked to a very high incident angle $\left(60^{\circ}\right)$ on the first surface of the small lenses. Zemax does not take into account Fresnel losses while calculating the relative illumination.

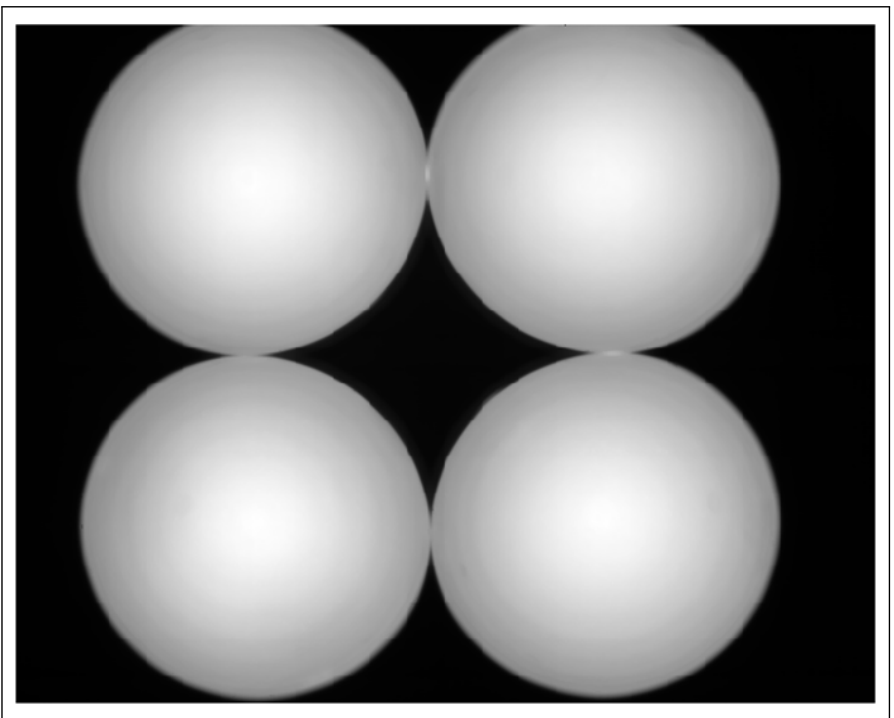

Fig. 8. Image acquired while illuminating the camera with a flat blackbody at $293 K$.

\section{B. Distortion measurement}

In order to measure the distortion of the cryogenic infrared camera, a resolved point source is scanned with a regular step within the field of view of the camera. It can be localized precisely on the detector by calculating its barycentre for each position. This measurement is performed simultaneously on the four channels of the camera. The relative position of the point sources in the FOV is calculated with respect to the optical centre of each channel. The four measured distortion cartographies are superimposed in Figure 10 and compared to the theoretical data provided by the Zemax software. The distortion is measured on a FOV equal to $69.8^{\circ} \times 84.54^{\circ}$. The amount of distortion $D$ is calculated as follows:

$$
D=\frac{\sqrt{\left(x_{r}-x_{p}\right)^{2}+\left(y_{r}-y_{p}\right)^{2}}}{\sqrt{x_{p}^{2}+y_{p}^{2}}},
$$

where $\left(x_{p}, y_{p}\right)$ are the coordinates of an image point of a system without distortion (black triangles in Figure 10(a)) and $\left(x_{r}, y_{r}\right)$ are the coordinates of an image point produced by the camera (color symbols in Figure 10(a)). In order to measure the maximum amount of distortion, we consider the coordinates of the extreme points at the corners. The maximal distortion is equal to $29.9 \%$ in the top right hand corner of the cartography, $30 \%$ in the bottom right hand corner, $31.1 \%$ in the bottom left hand corner, and $31.3 \%$ in the top left hand corner, whereas the maximal distortion calculated by Zemax is equal to $27.4 \%$ for this field of view. Therefore, the measured amount of distortion fits correctly the theoretical data.

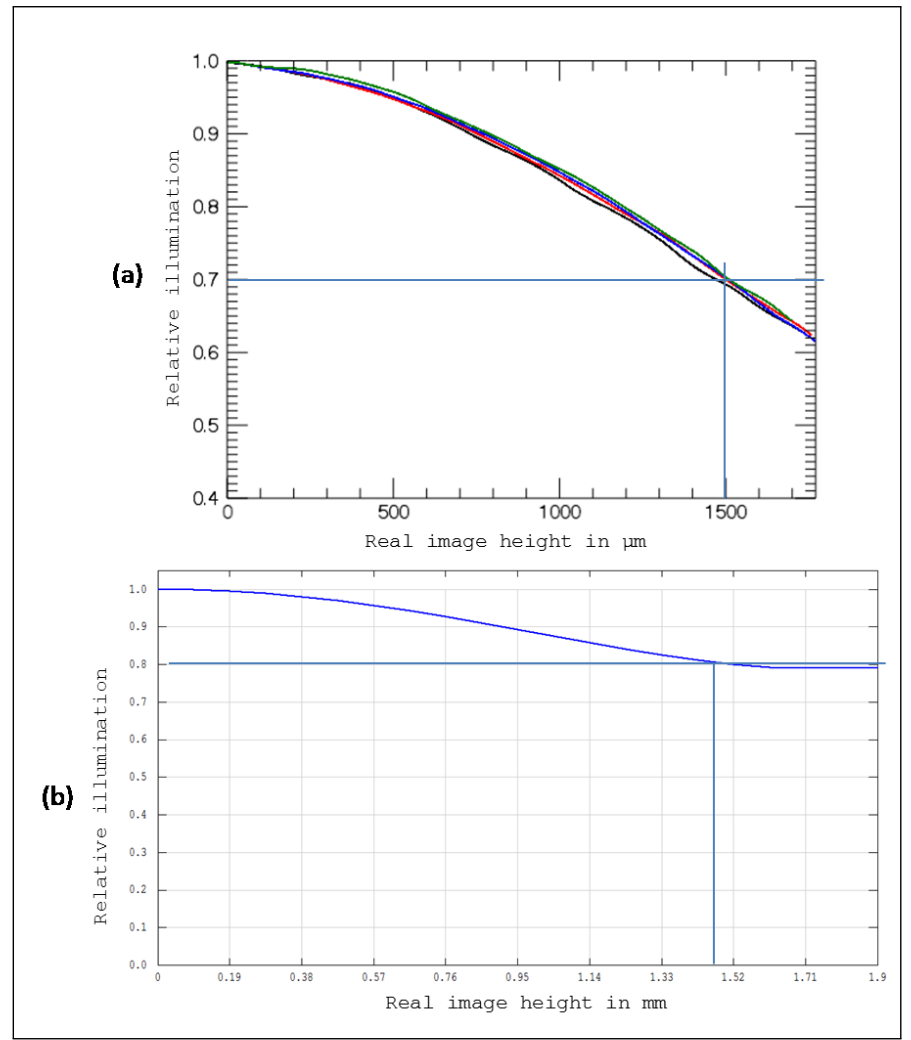

Fig. 9. Relative illumination as a function of height in the image plane, (a) measured data on the four channels (each colour corresponds to a channel), (b) theoretical data extracted from the Zemax software. 


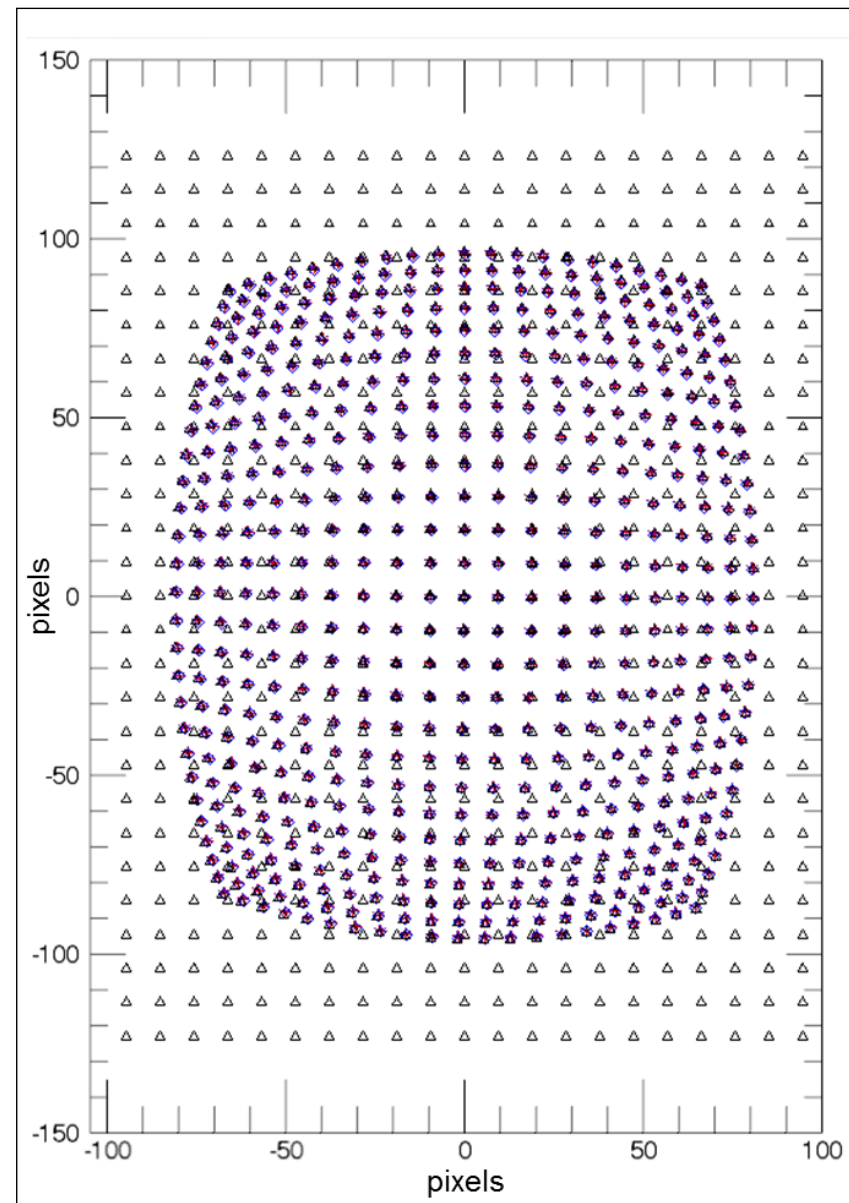

(a)

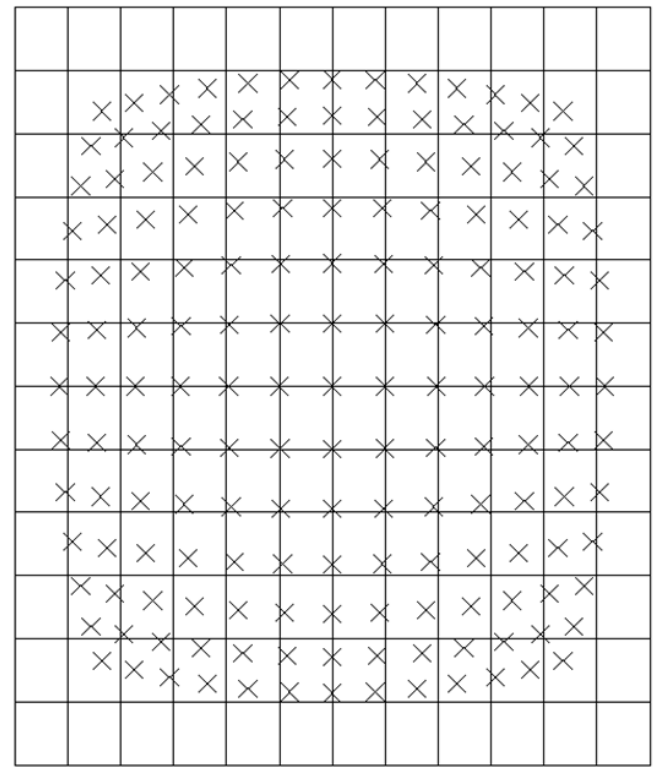

(b)

Fig. 10. (a) Superposition of the distortion cartographies measured in the four optical channels (in color) and a regular grid (black triangles), (b) Theoretical distortion data provided by the Zemax software.

\section{Discussion on tolerances and regular sampling of the scene}

In Figure 10, the distortion cartographies seem to be globally identical for the four optical channels. However, zooms on several areas for different FOV values highlight that the distortion slightly differs within the FOV, as illustrated in Figure 11. These differences may have the following explanations. The tolerance on the pitch between the channels, and the tilt between the lenslet array and the detector imply that the sampling on the optical axis is not the regular one expected from the theory. Moreover, optical aberrations are not identical for the four channels, since each channel has been machined independently by diamond turning: the sampling of the scene differs over the FOV (cf. Fig. 11(b)). Therefore, we have to use a more complex algorithm (called SR Viewer and presented in section 5) than a basic shift-and-add technique which may be employed as a first intention.

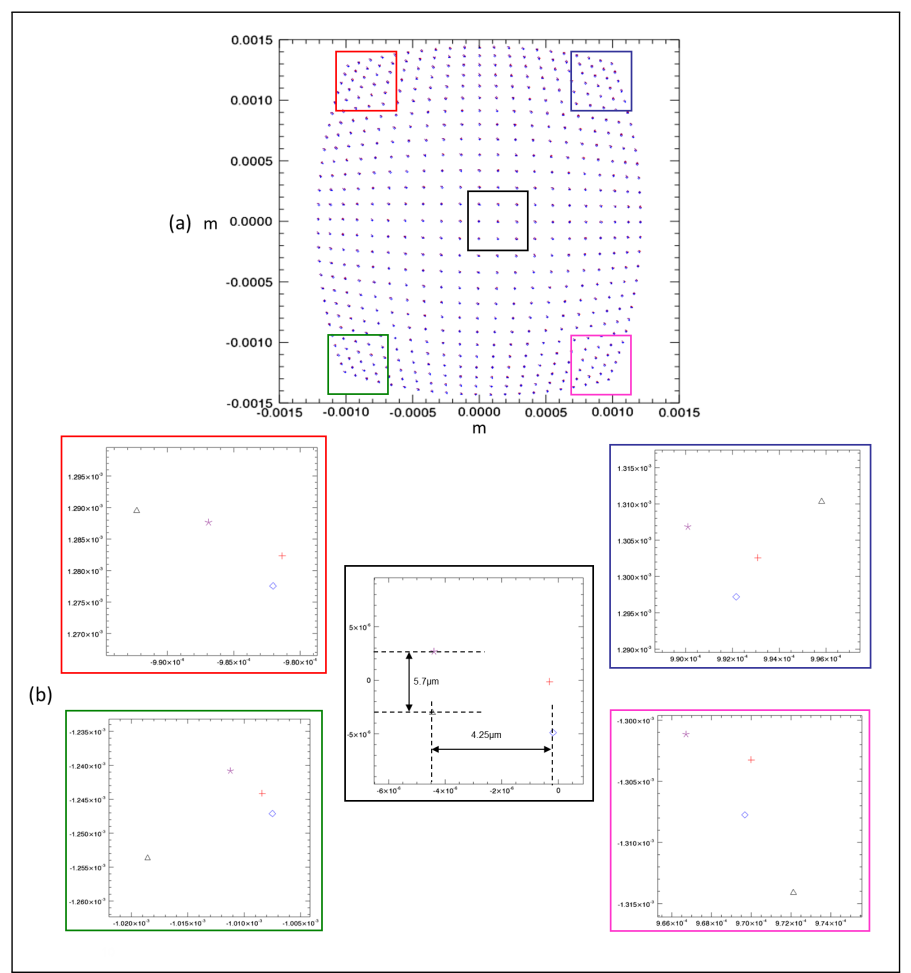

Fig. 11. (a) Reproduction of Fig. 10(a) with squares of different colors selecting different FOV, (b) Zooms on differences between the four optical channels for different FOV.

This prototype highlights that the tolerance on the tilt between the lenslet array and the detector has to be excessively tight in order to obtain the regular theoretical sampling of the scene. In Figure 12, we plot how the sampling varies with the tilt between the lenslet array and the detector.

As soon as a small tilt angle is introduced, the sampling differs from the regular one. We can even see that for a tilt angle of $0.11^{\circ}$, two of the four channels become redundant, which means that the diversity between the channels is not sufficient to enable the reconstruction of a higher resolution image. For our prototype, we measured a tilt angle equal to $0.46^{\circ}$. The theoretical sampling corresponding to a tilt angle of $0.46^{\circ}$ is illustrated in Figure 12(d). Moreover, there is also a tolerance on the pitch between the channels. This means that, in most cases, the 


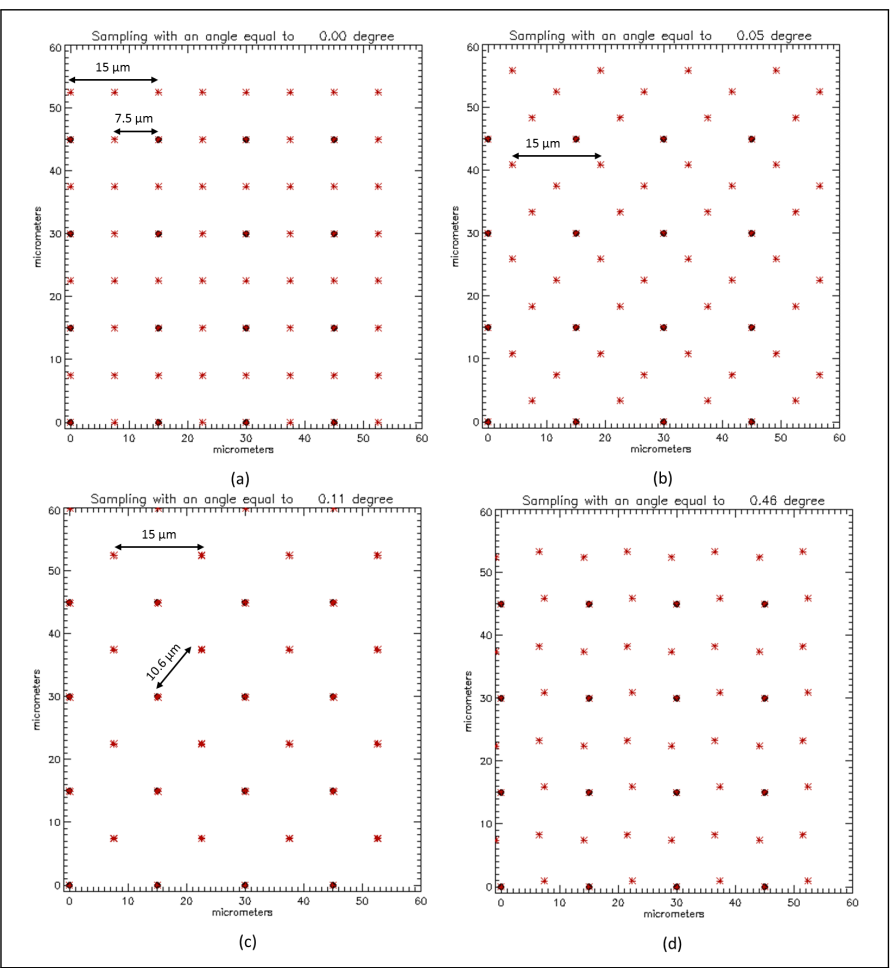

Fig. 12. Variation of sampling in the reconstructed image with the tilt angle, (a) theoretical case: tilt angle $=0^{\circ}$ and regular sampling, (b) tilt angle $=0.05^{\circ}$ and irregular sampling, (c) tilt angle $=0.11^{\circ}$ and 2 redundant subimages, (d) tilt angle $=0.46^{\circ}$ (case of our prototype).

sampling obtained with multichannel systems will be irregular.

To sum up, in practise, it is almost impossible to obtain a regular sampling of the scene because of the tolerances of optical components. To our knowledge, this has never been mentioned in literature on multichannel optical systems. This irregular sampling over the FOV makes the basic "shift and add" algorithm inefficient, and a more complex processing has to be employed.

\section{IMAGE RECONSTRUCTION}

\section{A. Elements on image reconstruction algorithm SR Viewer}

We have developed a super-resolution algorithm, called SR Viewer, which combines the differences between the subimages to retrieve spatial frequencies higher than the Nyquist frequency of a detector with a sampling pitch equal to $15 \mu \mathrm{m}$ $(33.3 \mathrm{cy} / \mathrm{mm})$.

SR Viewer has been used to process all the subimages of a single frame in order to yield a super-resolved image. It is based on minimization of the following criterion involving a term which matches the experimental data plus a regularization term:

$J\left(I^{H R}\right)=\sum_{m} \phi_{d}\left(I_{m}^{L R}-D W_{m} B I^{H R}\right)+\lambda\left(\phi_{r}\left(\nabla_{x} I^{H R}\right)+\phi_{r}\left(\nabla_{y} I^{H R}\right)\right)$,

where $I_{m}^{L R}$ denotes one of the 4 observed subimages, $I^{H R}$ denotes the sought SR image whose dimensions are those of $I_{m}^{L R}$ multiplied by a SR factor $L$. The observed image model involves a warp matrix $W_{m}$ that models the parallax motion between $I_{m}^{L R}$ and $I_{1}^{L R}$. The latter sets the reference geometry: in other terms $W_{1}$ is equal to the identity matrix. In practice, $W_{m}$ is based on optical flow estimates provided by the fast algorithm eFOLKI [34]. Matrix $B$ is a 2D convolution operator that accounts for sensor blur, modeled as a Gaussian point spread function. Matrix $D$ is a subsampling operator by the factor $L$ on both dimensions. $\nabla_{x} I^{H R}$ (resp. $\nabla_{y} I^{H R}$ ) denotes first-order, non centered finite difference gradients along horizontal (resp. vertical) direction. Penalizing functions $\phi_{d}$ is the Huber function [35], while $\phi_{r}$ is the Fair function [35]. These functions are parametrized by a scale parameter $s$ : for values larger than $s$, $\phi_{r}$ and $\phi_{d}$ have a linear behavior, while it is quadratic for values lower than $s$. The use of such non quadratic functions enables robustness to motion estimation errors for the data term and edge preserving for the regularization term. The trade-off between data term and regularization is tuned by the regularization parameter $\lambda$. Criterion (2) relies on an approximate image model where motion is applied after blur, such a model is strictly valid only for translation motion, but applies in practice in wider contexts [36][37]. Optimization of convex criterion (2) is performed using a fixed-step gradient descent, in practice ten iterations are enough. All the steps involved are highly parallel and the overall algorithm SRviewer has been implemented on GPU architecture. The examples of Figures 13 and 14 have been processed in $130 \mathrm{~ms}$ on a Nvidia Quadro M2000M.

\section{B. Retrieval of spatial frequencies higher than the Nyquist fre- quency}

We imaged a scene in a laboratory setting; the distance between the camera and the scene is equal to $2.5 \mathrm{~m}$. The scene is composed of a target (illuminated by a flat blackbody at the temperature of $305 \mathrm{~K}$ ) with a diagonal orientation. We make a twopoint non-uniformity correction (NUC) on each raw image acquired by the camera. This NUC correction ensures to avoid non-uniformity response between the pixels, otherwise, it is an issue when we intend to combine images. Figure 13(a) shows one raw subimage. As the subimage is undersampled, we can see a checker-board pattern instead of the target diagonal bars. Figure 13(b) is an interpolation of subimage (a). As expected, a simple interpolation does not allow to retrieve information, since the checker-board pattern is still visible. Figure 13(c) is the image obtained after super-resolution with the information contained in the four optical channels of the camera. We retrieve interesting spatial frequencies since the bar targets appear. However, some defaults on the transition between black and white zones are still visible, this is due to the irregular sampling. Figure 13(d) is the image after super-resolution obtained with the information contained in the four optical channels of the camera and temporal acquisition as well. We add diversity of information by acquiring a temporal sequence of images while moving slightly the camera in front of the scene. With this additional temporal diversity, some of the defaults visible in Figure 13(c) are corrected. When the sampling is irregular, it is difficult to have a good image quality after super-resolution with only $2 \times 2$ channels. However, designing a $3 \times 3$ multichannel system to improve the robustness of the super-resolution algorithm results in a loss of resolved points. The solution is therefore to design a $2 \times 2$ multichannel system and to add diversity by acquiring a temporal image sequence. The temporal acquisition of images is four times faster than what could be done with a single channel camera.

In order to access higher spatial frequencies, we imaged the same scene with increased distances $D$ between the camera and 


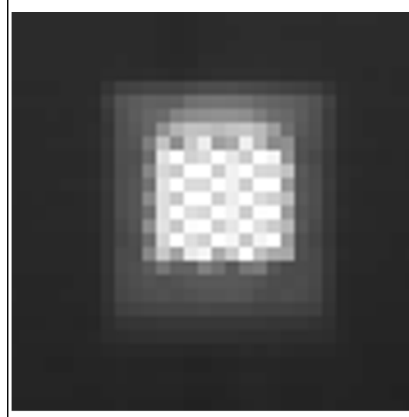

(a)

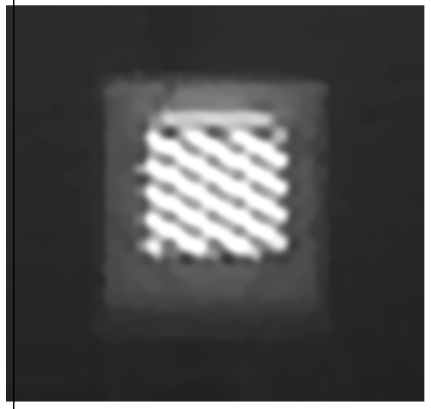

(c)

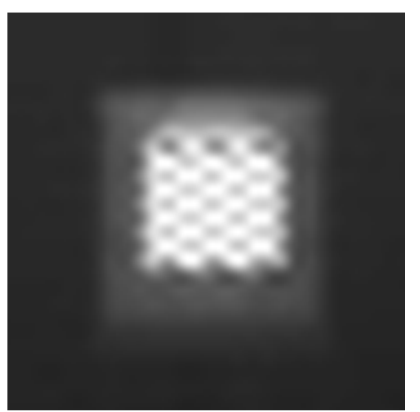

(b)

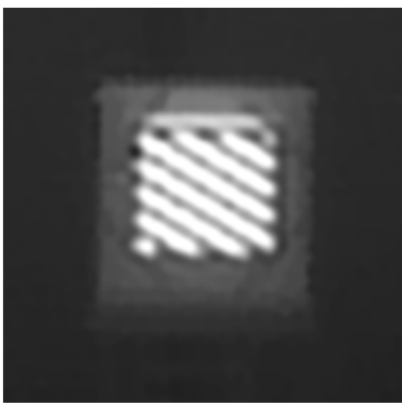

(d)
Fig. 13. (a) Raw subimage, (b) Interpolated subimage, (c) Image after super-resolution obtained with the information contained in the four optical channels of the camera, (d) Image after super-resolution obtained with the information contained in the four optical channels of the camera and temporal acquisition as well. the scene. The spatial frequency of the bar target is given by Equation 3 :

$$
v=\frac{N_{c y}}{L} \times D,
$$

where $v$ is the spatial frequency in cycles/radian, $N_{c y}$ is the number of cycles of the bar target, in our case, $N_{c y}=7, L$ is the dimension of the target (in $\mathrm{m}$ ), $D$ is the distance between the target and the camera. Therefore, when $D$ increases, it gives access to higher spatial frequencies. Images after processing are shown in Figure 14. Spatial frequencies higher than the Nyquist frequency can be retrieved with a satisfying contrast (for images (a), (b) and (c)). According to the measured MTF [30], the contrast goes to zero at $66 \mathrm{cy} / \mathrm{mm}$ (pixel cut-off frequency), then the contrast of the bar target is too low for images (d) and (e). We measured the bar target contrast on the images after image processing, and we compared it to the theoretical MTF given by Zemax (taking into account the filtering of the pixel) and to the experimental MTF measured with the spot scan method. This is illustrated in Figure 15 for spatial frequencies up to the cutoff frequency of the $15 \mu \mathrm{m}$ pixel pitch. We can see that the contrast measured on the bar target images clearly fits the MTF of the system. It clearly highlights contrasts higher than 0 for spatial frequencies greater than the Nyquist frequency. The optical quality of the multichannel system is much better than the one of its single channel equivalent (shown in Fig. 2). The contrast goes to zero at the cut-off frequency of the pixel.

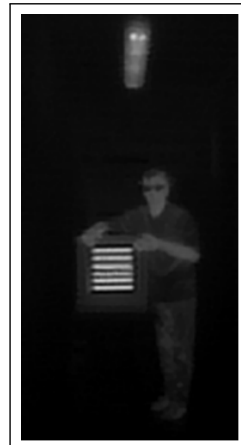

(a)

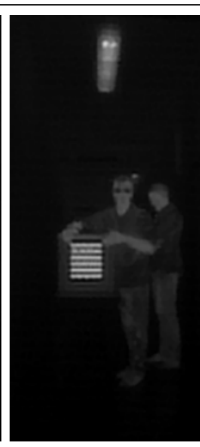

(b)

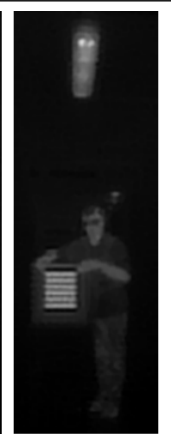

(c)

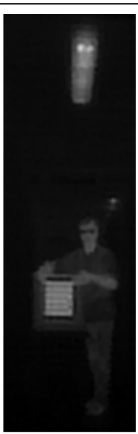

(d)

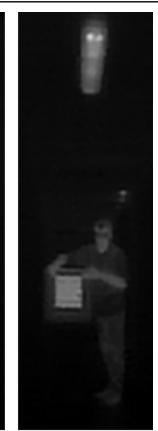

(e)
Fig. 14. Image of a scene in a laboratory setting for various distances $D$ between the camera and the scene, corresponding to various spatial frequencies: (a) $D=4 m(40 \mathrm{cy} / \mathrm{mm})$, (b) $D=4.5 m(44 c y / m m),($ c) $D=5 m(49 c y / m m),(d) D=5.5 m$ $(54 \mathrm{cy} / \mathrm{mm})$ and $(\mathrm{e}) \mathrm{D}=6 \mathrm{~m}(59 \mathrm{cy} / \mathrm{mm})$.

\section{CONCLUSION}

We have designed, manufactured and characterized an ultracompact cryogenic multichannel infrared camera, working in the $[3.7 \mu \mathrm{m}-4.8 \mu \mathrm{m}]$ spectral range. It is associated with a standard SCORPIO detector and integrated in a DDCA commercialized by SOFRADIR. The total track length of the optical module is lower than $6 \mathrm{~mm}$ only and the optical elements are directly integrated in the cold shield. The cool-down time is comparable to the one of an equivalent DDCA without an imagery function, because in the way we miniaturized the optical system, the additional optical and mechanical mass to be cooled is very low. The camera has a wide FOV of $120^{\circ}$, and is thus well suited for 


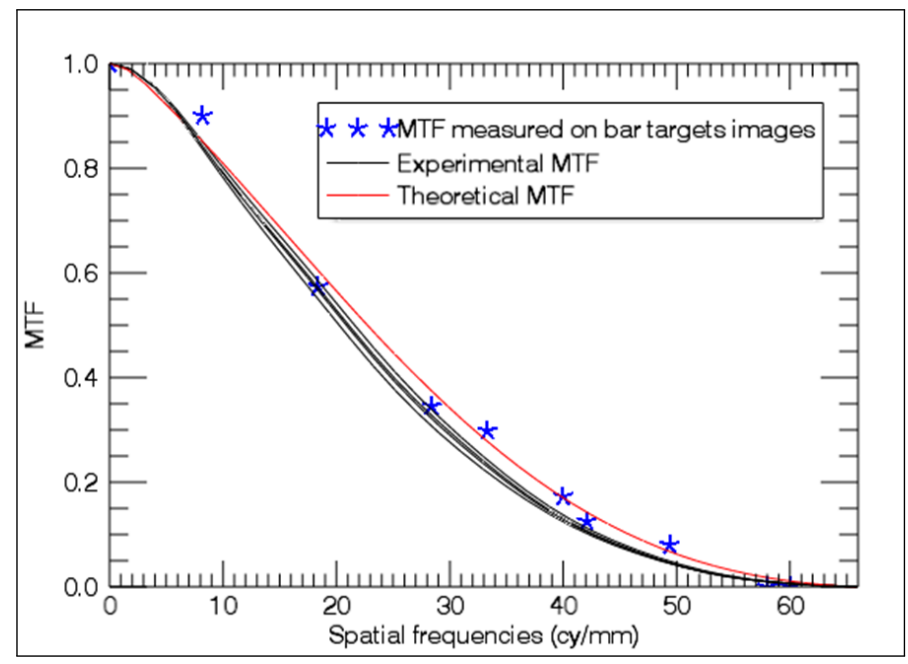

Fig. 15. MTF measured on the bar targets images: it fits well the theoretical and experimental MTF curves.

surveillance applications. In the MWIR band, due to the diffusion length of carriers in $\mathrm{HgCdTe}$, it is not worth trying to retrieve spatial frequencies higher that the cut-off frequency of the pixel (twice the Nyquist frequency). $2 \times 2$ channels are only necessary; adding more channels would result in a loss of resolved points in the final image, that is why we prefer keeping the highest number of resolved points by limiting the number of optical channels. Tolerances on the optical system lead to irregular shifts between the subimages. That is why the algorithm has to be more complex than a basic shift-and-add technique. Some redundancy exists between the four channels, and it becomes necessary to make temporal acquisitions of images to improve image quality after super-resolution. The optical quality of the channels is satisfying and fits the theoretical data. Cooling the optical system provides a very low NETD compared to traditional infrared cameras whose optical parts are outside the Dewar. These developments show that integrating optical functions near the IRFPA can be fully compatible with industrial and operational constraints. Within future work, associating an IRFPA with an array of lenses could be used to build more complex architectures in order to address various applications such as multispectral imaging or 3D vision.

This work was sponsored by the Defense Procurement Agency (DGA) of the French Ministry of Defense.

\section{REFERENCES}

1. A. W. Lohmann, "Scaling laws for lens systems," Appl. Opt. 28, 49964998 (1989).

2. R. Völkel, M. Eisner, K.J. Weible "Miniaturized imaging systems," Microelec. Eng. 67-68, 461-472 (2003).

3. J. Tanida, T. Kumagai, K. Yamada, S. Miyatake, K. Ishida, T. Morimoto, N. Kondou, D. Miyazaki and Y. Ichioka, "Thin observation module by bound optics (TOMBO): concept and experimental verification," Appl. Opt. 40, 1806-1813 (2001).

4. M. Shankar, R. Willett, N. Pitsianis, T. Schulz, R. Gibbons, R. Te Kolste, J. Carriere, C. Chen, D. Prather and D. Brady, "Thin infrared imaging systems through multichannel sampling," Appl. Opt. 47, B1-B10 (2008).

5. A. Portnoy, N. Pitsianis, X. Sun, D. Brady, R. Gibbons, A. Silver, R. Te Kolste, C. Chen, T. Dillon, and D. Prather, "Design and charac- terization of thin multiple aperture infrared cameras," Appl. Opt. 48 , 2115-2126 (2009).

6. K. Venkataraman, D. Lelescu, J. Duparré, A. McMahon, G. Molina, P. Chatterjee, R. Mullis, and S. Nayar, "PiCam: an ultra-thin high performance monolithic camera array," ACM Trans. Graph. 32, 166 (2013).

7. G. Carles, G. Muyo, N. Bustin, A. Wood, and A. R. Harvey, "Compact multi-aperture imaging with high angular resolution," JOSA A 32, 411419 (2015).

8. A. Papoulis, "Generalized sampling expansion," IEEE Trans. Circuits Syst. 24 (IEEE,1977), p. 652.

9. S. C. Park, M. K. Park, and M. G. Kang, "Super-resolution image reconstruction: a technical overview," IEEE Signal Process. Mag. 20(3), 21-36 (2003).

10. Y. Kitamura, R. Shogenji, K. Yamada, S. Miyatake, M. Miyamoto, T. Morimoto, Y. Masaki, N. Kondou, D. Miyazaki, J. Tanida and Y. Ichioka, "Reconstruction of a high-resolution image on a compound-eye imagecapturing system," Appl. Opt. 43, 1719-1727 (2004).

11. K. Nitta, R. Shogenji, S. Miyatake and J. Tanida, "Image reconstruction for thin observation module by bound optics by using the iterative backprojection method," Appl. Opt. 45, 2893-2900 (2006).

12. K. Choi and T. J. Schulz, "Signal-processing approaches for imageresolution restoration for TOMBO imagery," Appl. Opt. 47, B104-B116 (2008).

13. A. V. Kanaev, J. R. Ackerman, E. F. Fleet, and D. A. Scribner, "TOMBO sensor with scene-independent superresolution processing," Opt. Lett. 32, 2855-2857 (2007).

14. J. Shi, S. E. Reichenbach, and J. D. Howe, "Small-kernel superresolution methods for microscanning imaging systems," Appl. Opt. 45, 1203-1214 (2006).

15. J. Duparré, P. Dannberg, P. Schreiber, A. Bräuer and A. Tünnermann, "Artificial apposition compound eye fabricated by micro-optics technology," Appl. Opt. 43, 4303-4310 (2004).

16. J. Duparré, P. Schreiber, A. Matthes, E.Pshenay-Severin, A. Bräuer and A. Tünnermann, "Microoptical telescope compound eye", Opt. Express 13, 889-903 (2005).

17. J. Duparré, P. Dannberg, P. Schreiber, A. Bräuer and A. Tünnermann, "Thin compound-eye camera," Appl. Opt. 44, 2949-2956 (2005).

18. K. Stollberg, A. Brückner, J. Duparré, P. Dannberg, A. Bräuer, and A. Tünnermann, "The Gabor superlens as an alternative wafer-level camera approach inspired by superposition compound eyes of nocturnal insects," Opt. Express 17, 15747-15759 (2009).

19. G. Druart, N. Guérineau, R. Haïdar, S. Thétas, J. Taboury, S. Rommeluère, J. Primot and M. Fendler, "Demonstration of an infrared microcamera inspired by Xenos Peckii vision," Appl. Opt. 48, 3368-3374 (2009).

20. A. Brückner, J. Duparré, F. Wippermann, R. Leitel, P. Dannberg and A. Bräuer, "Ultra-compact close-up microoptical imaging system," Proc. SPIE 7786, 77860A-1 - 77860A-8 (2010).

21. A. Brückner, J. Duparré, R. Leitel, P. Dannberg, A. Bräuer, and A. Tünnermann, "Thin wafer-level camera lenses inspired by insect compound eyes," Opt. Express 18, 24379-24394 (2010).

22. J. Meyer, A. Brückner, R. Leitel, P. Dannberg, A. Bräuer, and A. Tünnermann, "Optical Cluster Eye fabricated on wafer-level," Opt. Express 19, 17506-17519 (2011).

23. L. Li, and A. Y. Yi, "Design and fabrication of a freeform microlens array for a compact large-field-of-view compound-eye camera," Appl. Opt., 51, 1843-1852 (2012).

24. L.C. Laycock and V.A. Handerek, "Multi-aperture imaging device for airborne platforms," Proc. SPIE 6737, 673709 (2007).

25. L.C. Laycock and V.A. Handerek, "Miniature imaging devices for airborne platforms," Proc. SPIE 7113,71130M-1 (2008).

26. G. Druart, F. de la Barrière, N. Guérineau, J. Deschamps, M. Fendler, N. Lhermet, J. Rullière, S. Magli, Y. Reibel, and J-B. Moullec, "Towards infrared DDCA with an imaging function," Proc. SPIE 8012, 801228-1 (2011).

27. F. de la Barrière, G. Druart, N. Guérineau, and J. Taboury, "Design strategies to simplify and miniaturize imaging systems," Appl. Opt. 50, 943-951 (2011). 
28. F. de la Barrière, G. Druart, N. Guérineau, G. Lasfargues, M. Fendler, N. Lhermet, and J. Taboury, "Compact infrared cryogenic wafer-level camera: design and experimental validation," Appl. Opt. 51, 10491060 (2012).

29. http://www.sofradir.com.

30. F. de la Barrière, G. Druart, N. Guérineau, M. Chambon, A. Plyer, G. Lasfargues, E. de Borniol, and S. Magli, "Development of an infrared ultra-compact multichannel camera integrated in a SOFRADIR's Detector Dewar Cooler Assembly," Proc. SPIE 9451, 94510E-1-94510E10 (2015).

31. J. Berthoz, R. Grille, L. Rubaldo, O. Gravrand, A. Kerlain, N. PereLaperne, L. Martineau, F. Chabuel, and D. Leclercq, "Modeling and characterization of MTF and spectral response at small pixel pitch on Mercury Cadmium Telluride," Journal of Electronic Materials 44, 31573162 (2015)

32. T. Grulois, G. Druart, H. Sauer, M. Chambon, N. Guérineau, S. Magli, G. Lasfargues, and P. Chavel, "Reduction of material mass of optical component in cryogenic camera by using high-order Fresnel lens on a thin germanium substrate," Appl. Opt., 54, 6313-6320 (2015).

33. http://www.savimex.fr

34. A. Plyer, G. Le Besnerais, and F. Champagnat, "Massively parallel Lucas Kanade optical flow for real-time video processing applications," Journal of Real-Time Image Processing, 1-18 (2014).

35. W. Rey, Introduction to Robust and Quasi-Robust Statistical Methods, Springer-Verlag Berlin Heidelberg (1983).

36. A. Létienne, F. Champagnat, G. Le Besnerais, C. Kulcsár, and P. Viaris De Lesegno, "Fast Super-Resolution on Moving Objects in Video Sequences," in EUSIPCO European Signal Processing Conference (2008).

37. R. C. Hardie, "A Fast Image Super-Resolution Algorithm Using an Adaptive Wiener Filter," IEEE, 16 2953-2964 (2007). 